\title{
Rôle de la végétation dans les processus de propagation de la sécheresse dans les sols argileux
}

T. TESSIER

Physico-chimie et écotoxicologie des sols INRA Versailles tessier@versailles.inra.fr

\section{Y. COQUET}

UMR INRA/AgroParisTech Environnement et Grandes Cultures BP 01-78850

Thiveral-Grignon Yves.Coquet@agroparistech.fr

\section{Y. LEFÈVRE, N. BRÉDA} UMR INRA-UHP 1137 Écologie et Écophysiologie forestières F-54280 Champenoux breda@nancy.inra.fr, lefevre@nancy.inra.fr
La végétation constitue la source majeure de prélèvement naturel d'eau du sol. Elle contribue à l'installation puis à la propagation de la sécheresse en profondeur dans les sols. Ses capacités d'extraction de l'eau du sol sont liées à son système racinaire, dont l'extension verticale peut facilement coloniser des horizons argileux, présentant souvent une forte teneur en eau et constituant une ressource de survie lors de sécheresses extrêmes, en particulier pour les arbres. En effet, de nombreuses espèces ligneuses sont capables d'extraire l'eau du sol à des potentiels très inférieurs au potentiel au point de flétrissement permanent des cultures annuelles. Le dessèchement des sols sous végétation peut alors entraîner des déformations du sol, le plus souvent réversibles après retour des pluies. En 2003 toutefois, la sécheresse atteinte sur certains sols argileux, en particulier sous les arbres, a pu induire des déformations difficilement réversibles, pouvant conduire à long terme à des évolutions de la densité apparente du sol.

Mots clés : sécheresse, racine, sol argileux, retrait/ gonflement, forêt, prairie, culture.

\section{Role of vegetation in the phenome- non of drought propagation within clayey soils}

The vegetation is the main source of water uptake from soils. It contributes to drought propagation within the soil. The ability of vegetation to extract water is due to its root system, whose vertical distribution may reach clayey soil layers. Most of the time, these clay layers have high soil water content and can be considered as a water storage for survival during extreme drought event. This is especially true for trees which are able to extract water at potentials lower than that at permanent wilting point for annual crops. Soil under vegetation can then reach such low soil water potential that soil shrinkage occurs. This phenomenon is generally reversible when soil water content is recovered. Nevertheless, during the 2003 drought event in Europe, clay soils below trees dehydrated so much that notfully-reversible deformations could occur, possibly leading to long term evolution of soil bulk density.

Key words: drought, roots, clay soil, forest, crop, soil shrinkage. 


\section{Introduction}

Les récentes sécheresses que la France a connues rappellent que des précipitations insuffisantes pendant une période prolongée conduisent à des déficits en eau qui affectent la végétation et les sols. Les interactions entre le sol, la végétation et l'atmosphère sont complexes. La végétation intervient sur : 1) la redistribution et l'évaporation des précipitations qu'elle intercepte: 2) l'extraction de l'eau par le systeme racinaire; 3) le contrôle biophysique de la transpiralion. Ainsi, la végétation est la principale source naturelle de modification de la teneur en eau des sols. En retour, la teneur en eau du sol contraint l'évapotranspiration de la végétation, en imposant un ajustement de la consommation en eau par fermeture des stomates en fonction de la disponibilité en eau du sol.

La sécheresse édaphique peut être définie comme une période pendant lacuelle la quantité d'eau disponible dans le sol est insuffisante pour permetre le développement, la croissance et le fonctionement optimal de la végétation. C'est donc par la mesure ou la modélisation de la teneur en eau du sol qu'il est possible de caractériser et quantifier les épisodes de sécheresse édaphique. Sous un climat donné, un sol se dessèchera plus ou moins rapidement et profondément selon qu'il est nu, occupé par de la végétation de type herbacée ou ligneuse. Comprendre et estimer l'impact de la végétation sur la dynamique de l'eau dans le sol est essentiel pour pourvoir prédire la propagation spatio-temporelle de la sécheresse dans le sol et les déformations physiques possibles qui peuvent en résulter.

\section{2}

\section{Le sol, un milieu hétérogène}

Le sol, ou couverture pédologique, est un objet naturel dont l'existence et l'état actuel résulte d'une évolution au cours du temps d'un matêriau géologique sous l'action combinée de facteurs climatiques (précipitations, température) et de facteurs biotiques (animaux et végétaux, microorganismes). Ces différentes actions conduisent à la formation d'un ensemble d'horizons issus de l'altération du matériau originel at de l'incorporation de matière organique. Ces phénomènes créent la porosité (fissurale, texturale, structuralel, le milieu s*organise, 1’aération et la perméabilité augmentent: Les transferts hydriques et gazeux sont favorisés, ainsi que l'activité biologique.

Des conditions du milieu favorables tendent à augmenter lá fourniture d'eau à la plante et améliore sa croissance, et augmente indirectement le retrait/gonflement par une plus grande consommation en eau qui amplifie l'alternance saisonnière des cycles dessêchement - réhydratation.

La vẻgétation intervient dans deux flux opposés du bilan d'eau du réservoir que constitue le sol: d'une part, la végétation réduit et retarde l’entrée d'eau en interceptant les précipitations; d'autre part, elle intervient directement sur la sortie d'eau, en pusant l'eaw nécessaire à sa transpiration. L'évapotranspiration du couvert végétal (transpiration plus évaporation de l'eau interceptée sur le feuillage, qui peut être quantitativement importante sur les arbres) est concitionnée par la demande climatique, souvent caractérisée par l'évapotranspiration potentielle (ETP). Cette variable climatique intègre à la fois les termes radiatif (rayonnement) et convectif (vitesse du vent, humidité relative de l'air) qui contrôlent l'évaporation. La quanlité d'eau maximale prélevée par la végétation dans le réservoir du sol est fonction de cette ETP et de la surface foliaire du couvert. A certaines périodes de l'année, l'eau falblement retenue s'évacue en profondeur et conlribue à alimenter les réserves profondes (nappe phréatique). Si le sol est imperméable (horizon très argileux par exemple) et ne laisse pas l'eau s'infittrer, il y a alors formation d'une nappe temporaire (dite perchée), oú de l'eau libre s'accumule en saturant toute la porosité du sol au dessus du plancher imperméable. Là encore, le régime d'alternance nappe temporaire - sécheresse amplifie les phénomènes de retrait gontlement dans les sols sensibles à ces phénomènes.

Par définition, la réserve utile intrinsèque du sol est la quantité d'eau, exprimée en volume d'eau par volume de sol, comprise entre deux limites appelées états hydriques caractéristiques : la capacité au champ et le point de flétrissement permanent [Fig. 1]. La capacité au champ comespond à la quantitể maximale de rétention en eau du sol, après le ressiuyage de l'eau gravitaire. Ce niveau est généralement atteint en fin $d^{\prime} h i-$ ver, juste avant la reprise de la végétation. Cependant, il arrive, en particulier Iors de succession c'années à déficit pluviométrique, que cet état ne soit pas reconstitué chaque hiver. Le potentiel hydrique du sol à la capacité au champ est généralement considéré égal à $-0,01$ MPa. La seconde limite, appelée point de flétrissement permanent, correspond à la limite inférieure de teneur en eau en dessous de laquelle la végétation n'est plus capable d'absorber l'eau. Cet état hydrique caractéristique correspond à la teneur en eau du sol à un potentiel hydrique de $-1,6 \mathrm{MPa}$. Cette notion, d'origine agronomique, reste assez theorique, car certaines plantes dont de nombreux arbres sont capables d'extreire de l'eau à des potentiels encore plus négatifs. Pour un sol de texture donnée, la réserve utile intrinsèque correspond à la différence entre les teneurs en eau volumiques caractéristiques. La figure 1 montre qu"un sol de texture limoneuse ou de texture argileuse aura une meilleure réserve utile intrinsèque qu'un sol de texture sableuse. Cette réserve utile intrinséque est génêralenent exprimée en mm de hauteur d'eau par cm de profondeur de sol. La quantité d'eau effectivement disponible pour un couvert végétal, appelée simplement réserve utile, śobtient en multîliant lá réserve utile intrinsèque du sol par la profondeur d'enracinement effective du couvert.

La plus grande majorité des travaux sur la rétention de l'eau utilisent des relations empiriques permettant de prédire la teneur en eau d'une couche de sol à un de ces états caractéristiques. Ces relations, appelées fonctions de pédotransfert (Bastet et al., 1998), utilisent les propriétés physico-chimiques des sols comme prédicteurs et sont de type :

$W=a *$ Argile $+b *$ Limon $+c$ * Sable $+d *$ Corg $+e$ * Da avec:

W, teneur en eau massique;

Argile, teneur en argile;

Limon, teneur en limon ;

Sable, tenewr en sable;

Corg, teneur en carbone organique;

Da, densité apparente sèche ;

$\mathrm{a}, \mathrm{b}, \mathrm{c}$, d et $\mathrm{e}$, coefficients de calage. 


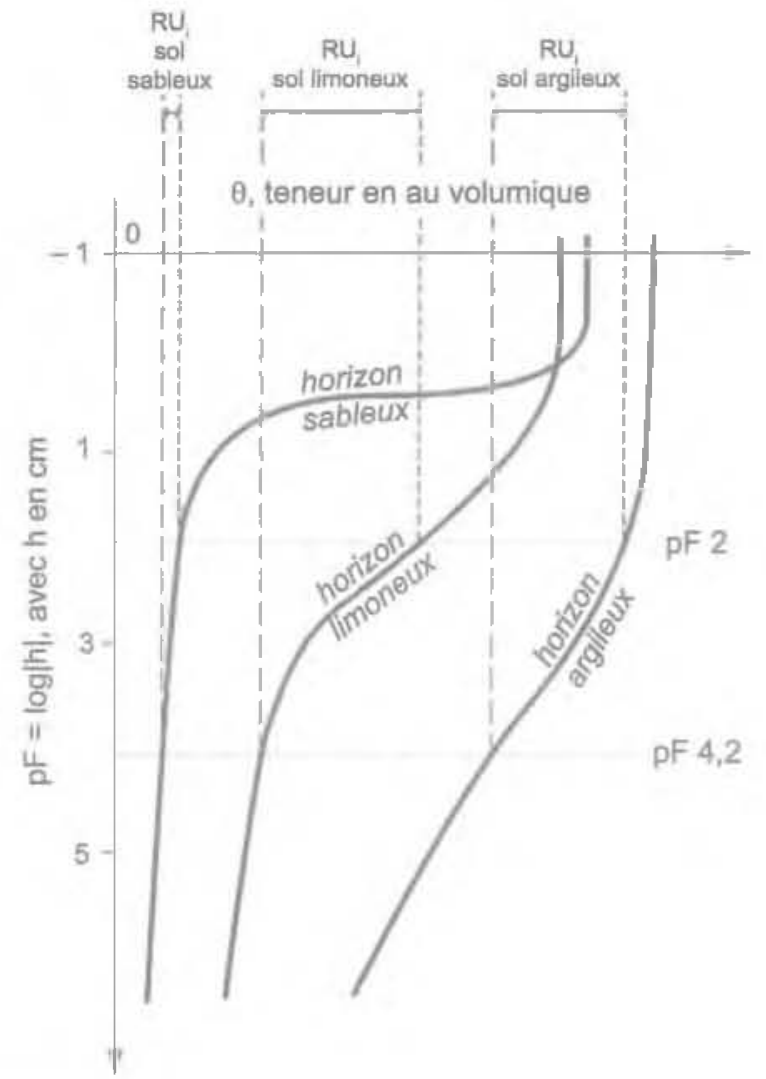

FG. I Représentation de la réserve utile instrinsèque (RU) comme la différence entre la teneur en eau à $-0,01 \mathrm{MPa}$ ( $\mathrm{pF} 2$ ), correspondant, par convention, à la capacité au champ, et la teneur en eau à $-1,6 \mathrm{MPa}(\mathrm{pF}$ $4,2)$, correspondant au point de flétrissement permanent de la plupart des plantes cultivées. La forme des courbes reliant le potentiel matriciel, $h$, à la teneur en eau, $\theta$, varie selon la texture. De manière générale, la $R U_{\text {, du }}$ sol limoneux est supérieure à celle du sol argileux et celle du sol sableux est très faible.

Cette fonction utilise des prédicteurs simples à apprécier sur le terrain (texture) ou assez facilement mesurables. Dans d’autres cas, les paramètres utilisés dans ces fonctions sont plus complexes à déterminer, mais mesurés en routine par les laboratoires d'analyse des sols. Par exemple, la figure 2 illustre un exemple de relation utilisable pour prédire la teneur en eau résiduelle (au point de flétrissement) à l'aide de la capacité d'échange cationique (d'après Tessier et al., 1996). D'autres exemples peuvent être retrouvés dans Bastet et al. (1998), dans Bruand et al. (2003, 2004) ou encore Cresswell et al. (2006).

\section{3}

\section{La végétation et ses caractéristiques}

La végétation est située à l'interface entre l'atmosphère et le sol. Son impact spatial et temporel sur la dynamique de l'eau dans le sol est directement conditionné par les caractéristiques de ses deux surfaces d'échanges : la surface foliaire, siège des échanges plante-atmosphère, et le système racinaire fin, slège des échanges plante-sol. L'interface couvert-atmosphère est caractérisẻe par sa phénologie (dynamique saisonnière, apparition et chute des feuilles, fanaison) et son indice foliaire. Ce paramètre exprime la sur-

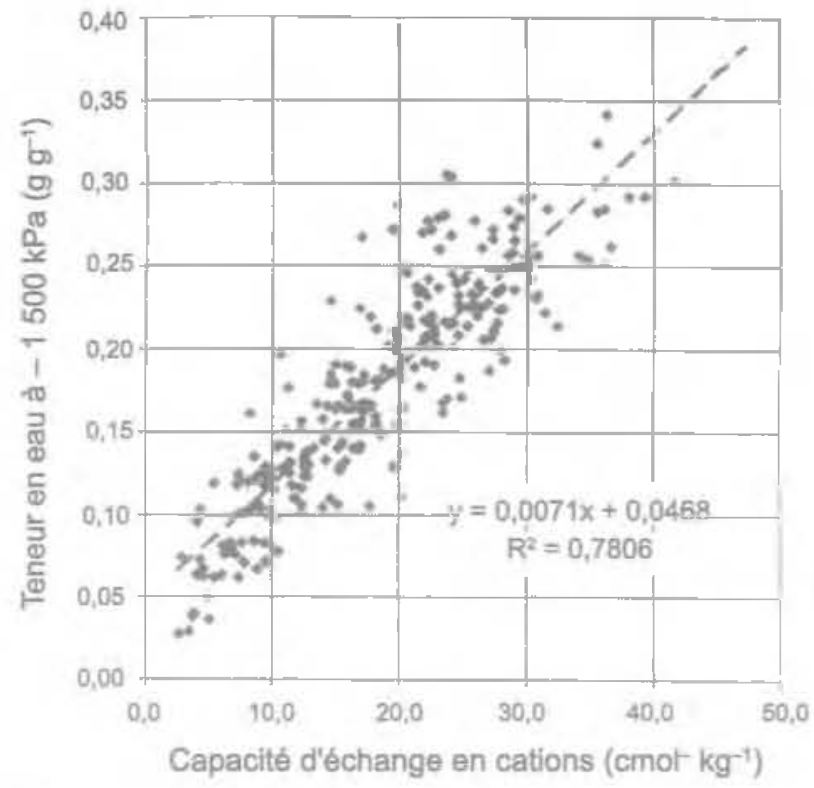

Fa. \& Prédiction de la teneur en eau résiduelle (au point de flétrissement) à l'aide de la capacité d'échange cationique (CEC) (d'après Tessier et al., 1996).

face de feuilles développée par unité de surface de sol. Ainsi, un indice foliare de 4 signifie qu'il y a $4 \mathrm{~m}^{2} \mathrm{de}$ feuilles au-dessus $\mathrm{d}^{\prime} 1 \mathrm{~m}^{2} \mathrm{de}$ sol. Cette grandeur peut être mesurée soit directement en récoltant les feuilles (en coupant une surface définie pour les couverts herbacés ou en récoltant les feuilles lors de leur chute automnalel, soit indirectement en mesurant le rayonnement transmis sous la végétation (Bréda, 1999). L'interface entre sol et plante est caractérisée quant à elie par la distribution verticale et horizontale des racines. Dans le cadre de l'étude du rôle de la végétation sur la propagation de la sécheresse du sol, seule la fraction des racines qui absorbent effectivement l'eau du sol, est à considérer. Cette remarque importe surtout pour les végétations pérennes et ligneuses, dont les systèmes racinaires les plus couramment décrits ne concernent que les racines d'ancrage, souvent plus superficielles et qui servent de support aux racines fines. En outre, les racines fines présentent un renouvellement et une croissance rapides, l'extension verticale des zones de prélèvement d'eau peut donc fortement évoluer au cours de la sajson.

\section{4}

\section{Quelle approche pour décrire la variation de teneur en eau du sol sous végétation ?}

Sous couvert d'arbres, les mesures conjointes de la consommation en eau des arbres (mesure du llux de sève brute dans le tronc) et de teneur en eau du sol renseignent quant à la propagation verticale du dessèchement au cours de la progresston de la sécheresse (Bréda et al, 1995). Sur l'exemple présenté figure 3 ; le prélèvement d'eau par des frênes a été estimé par différence de teneur en eau entre deux dates sans épisode pluvieux dans chaque couche de sol (Bréda et al, 2004). Parallèlement, la consommation a été mesurée par flux 
de sève dans les troncs des arbres au cours de chaque période et correspond au cumul des absorptions par couche de sol. Une fosse a été ouverte pour décrire la distribution verticale des racines, en distinguant les racines par classes de dimension. Au printernps, l'alimentation en eau par les arbres est assurée par le premier mètre d'épaisseur de sol. C'est dans cette couche de sol que toutes les racines pérennes ont étë observées et que la densité de racines finges est la plus importante. Au cours du mois de juin, le dessechement progresse en profondeur, et l'absorption d'eau est assurée dans une couche où seules des racines fines sont présentes. Dans cet exemple, cette couche capable de foumir de l'eau est à texture argileuse et présente une rétention en eau forte. Au cours de l'été, le premier mètre de sol ne foumit pratiquement plus d'eau. Les arbres mobilisent alors des réserves hydriques profondes, à l'aide des quelques pourcents de racines situées entre 1,2 m et $1.6 \mathrm{~m}$. On constate donc que les prélèvements ne sont pas proportionnels à la quaritité de racines fines tout au long de la saison. Des résultats comparables ont été obtenus sous chênaie (forêt de Champenoux, Bréda et al., 1995), avec une prospection efficace par les racines fines dans les horizons argileux. Les niveaux de dessèchement obtenus ont atteint des potentiels hydriques de $-2,5 \mathrm{MPa}$ dans l'horizon argilo-limoneux et de $-3,0 \mathrm{MPa}$ dans les horizons profonds (Fig. 4). Les horizons argilleux carbonatés comportent 70 à $80 \%$ d'un mélange smectite-vermiculite à forte potentialité de retrait gonflement. Ces deux exemples mettent en évidence l'importance de la profondeun, de la répartition et de la dynamigue du systeme racinaire.

\section{5}

\section{Exemples de bilans hydriques modé- lisés en fonction de la végétation}

Un premier exemple conceme la comparaison de ha dynamique d'eau dans le sol sous un couvert d'arbres à feuilles caduques (hêtre) et sous résineux à feuilles persistantes, avec un indice foliaire maximal comparable égal à 7,5 . Cette situation est tout ta fait réaliste et représente par exemple le cas d'une plantation résineuse

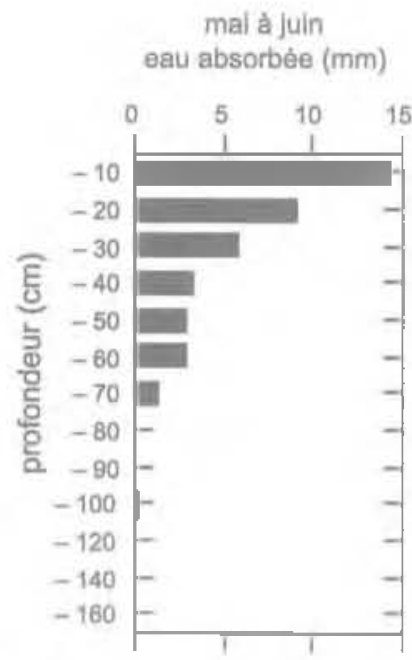

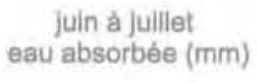
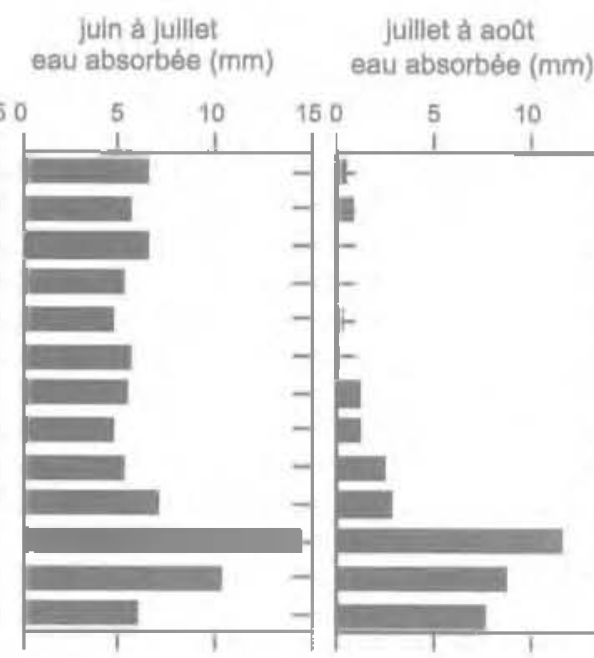

eau absorbée ( $\mathrm{mm})$

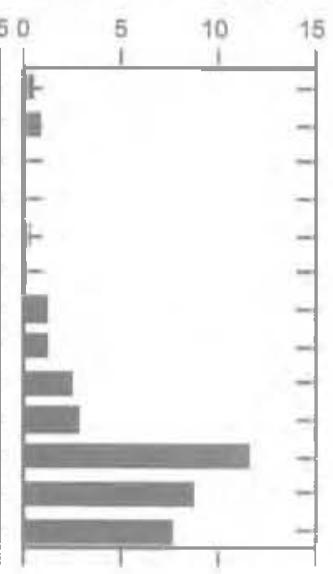

densité racinaire (racine/ $\mathrm{m}^{2}$ )

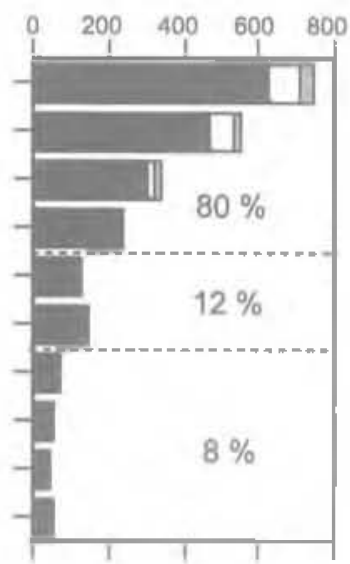

Progression en profondeur des prélèvements d'eau dans le sol au cours de l'établissement d'une sécheresse estivale naturelle sous un peuplement de frënes. L'eau absorbée est calculée à partìr des variations de teneur en eau du sol mesurées à la sonde à neutrons. La distribution verticale des racines est présentées en parallè̀le, en distinguant les racines par classes de dimensions (0-2 mm en noir, 2-5 mm en blanc, 5-10 mm et $>10$ mm en grisé). Les pourcentages de racines fines (0-2 mm) par horizon pédologique sont indiqués (d'après Bréda et al., 2002),

Profondeur

(cm)

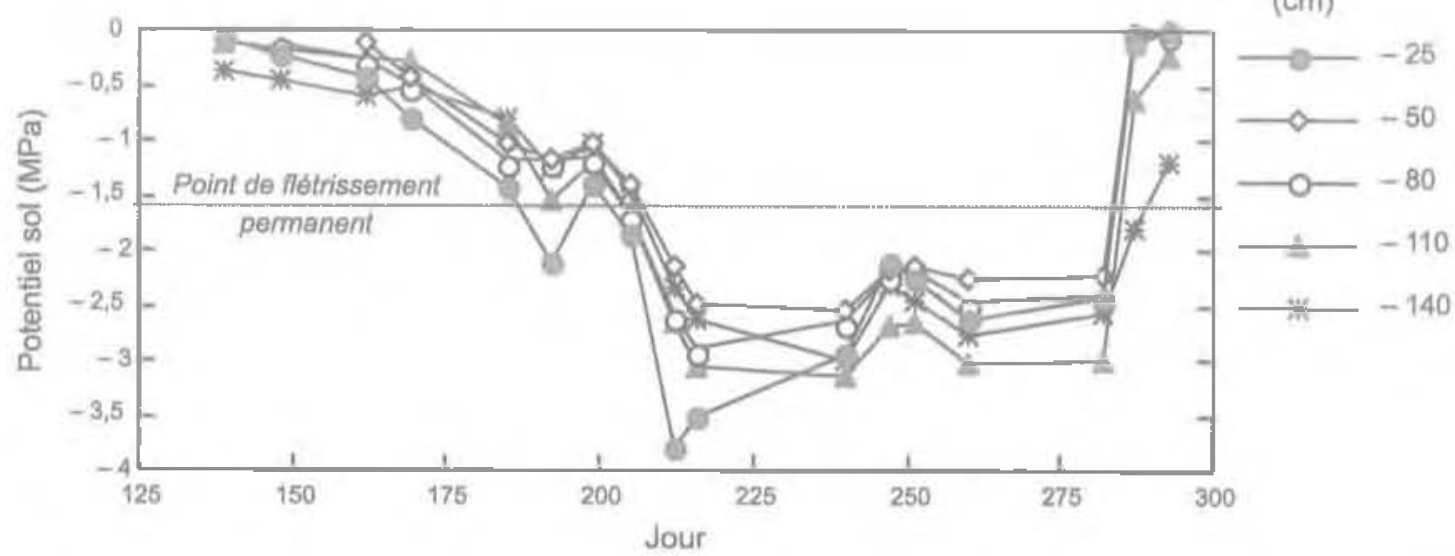

76. A Évolution du potentiel hydrique mesuré sous un sol forestier à 5 profondeurs à l'aide de mycropsychromètres au cours d'un dessèchement saisonnìer. Les potentiels mesurés dans le sol sont très nettement ìnférieurs au potentiel théorique correspondant au point de flétrissement permanent (ligne pointillée) (d'après Bréda et â., 1995), 
dense et une futale de hêtre, sur un sol limono-argileux sans contrainte majeure, d'une profondeur utile de $2 \mathrm{~m}$ pour les deux espèces, et présentant une réserve utile de $175 \mathrm{~mm}$. Les différences illustrées sur la figure 5 résultent de l'impact de la différence de phénologie (mise en place de toutes les feuilles au printemps sur feuillus). Cet effet de la phénologie est de bien plus grande importance que toutes les différences subtiles, telles que la régulation stomatique, qui jouenk à la marge dans ce schéma de fonctionmement. Le des. sèchement du sol au printemps est plus précoce sous les résineux, dont la consommation en eau débute dès que la température et le rayonnement augmentent. A l'opposé, la réhydratation du sol est retardée, en raison du maintien d'une forte interception des précipjtations lors du retour des pluies d'automne. Sur l'exemple de l’année 2003 en Lorraine, on peut calculer le début de la période de sécheresse au 28 mars sous résineux et au 16 juin sous feuillus (Brédá et al., 2004) : c'est la date à laquelle la réserve en eau chute en dessous de $40 \%$ de la réserve utile, limite représentée sur la figure 5. Pour les forêts, et ceci quelle que soit l'espèce, nous définissons en effet le déficit hydrique par rapport à ce seuil qui affecte à la fois la croissance, qui s'arrête, et la transpiration (régulation stomatique) (Granier et al., 1999). Dans la suite, le déficit hydrique que nous utiliserons correspond pour chaque courbe à la surface comprise entre ce seuil et la courbe d'évolution de la réserve en eau du sol. Ici cet indice est une grandeur sans dimension et est illustré par la zone hachurée en encart de la figure 5 . Ce type de calcul de bilan hydrique a ếté appliqué sur uné série cilmatique de 50 ans et permet d'établir un classement objectif des intensités de déficit hydrique entre les années, sous differents types de couvert végétal. In s'agit ici de quantifier l'impact d'un profil climatique sur la contrainte suble par couvert forestier donné, sans présager des ajustements ou réactions (de type dépérissement par exemple) qui pourraient s'en suivre. La figure 6 illustre ainsi le classement des 10 années à déficit hydrique
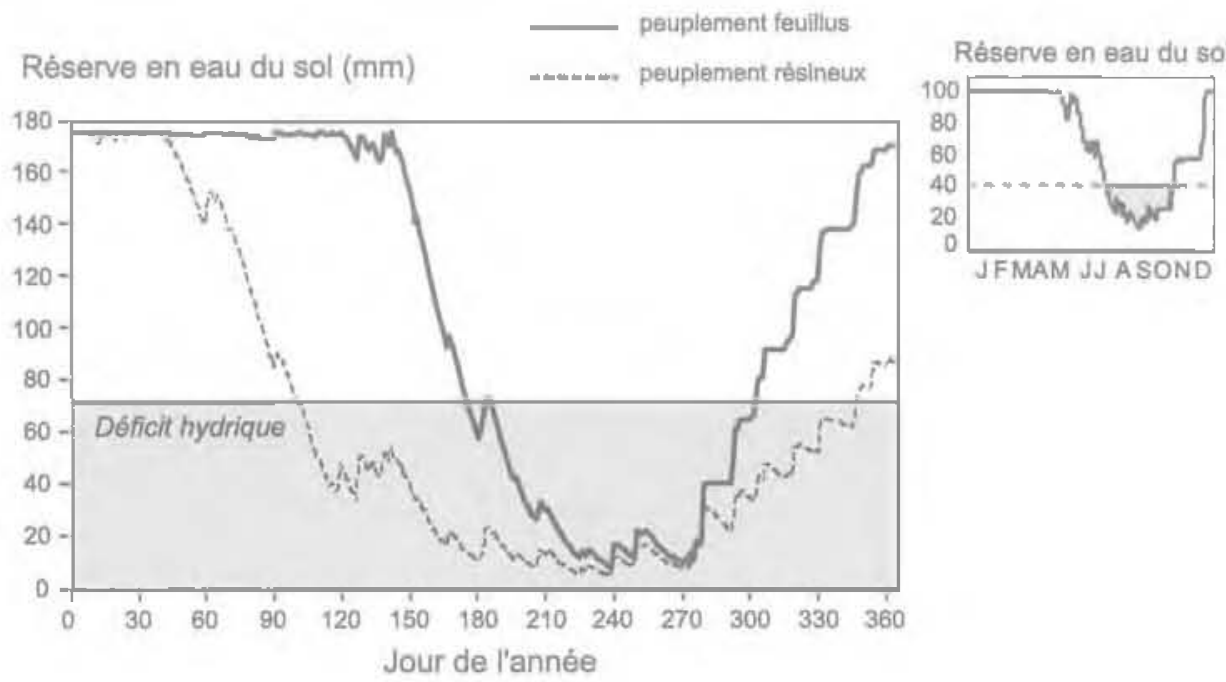

Evolution simulée de la réserve en eau d'un sol à forte réserve (175 mm) au cours de l'année 2003 à Nancy, sous peuplement feuillus ou résineux å indice foliaire équivalent $(7,5)$. Modélisation BLJOU (Granier et al., 1999), données climatiques INRA-Champenowx.

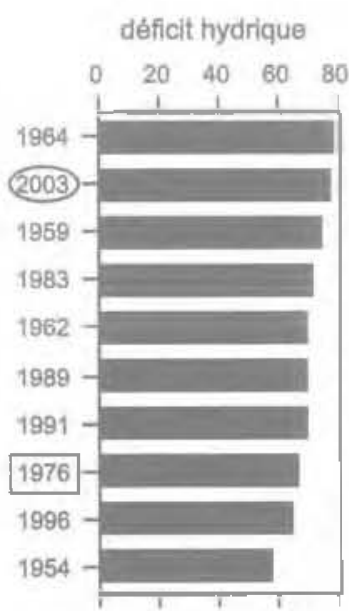

Peuplement feuillus

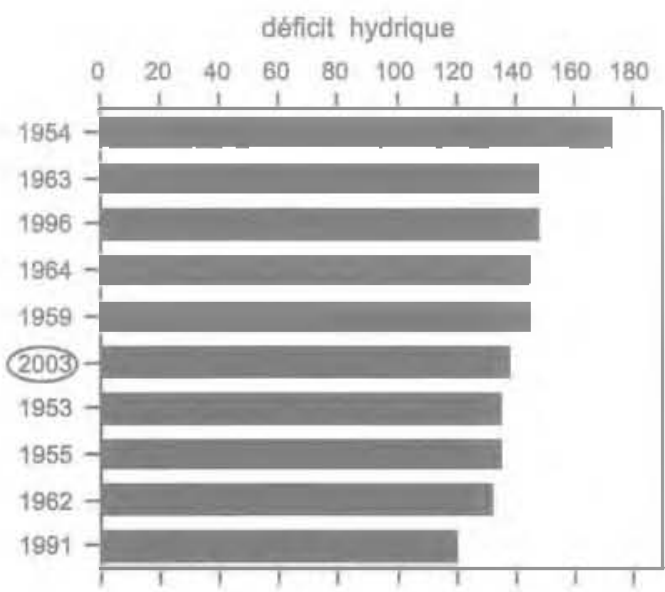

Peuplement résineux

M6. Classement des dix années à déficit hydrique les plus sévères par ordre décroissant d'intensité en Lorraine entre 1950 et 2003 , sous peuplement feuillus (a gauche) ou résineux (à droite) à indice foliaire égal est assez fort $(7,5)$, sur sol à réserve utile de $130 \mathrm{~mm}$. Les intensités des déficits hydriques sont environ deux fois plus intenses sous résineux que sous feuillus. L'année 2003 n'est pas la plus grave sous résineux des 50 dernières années et l'année 1976 n'est pas classée parmi les 10 années les plus sévères pour ces hypothèses et sous ce clímat. Sous feuillus, la sécheresse de l'arnée 1964 avait été légérement plus forte que celle de 2003. Modélisation BILJOL (Granier et al., 1999), données climatiques INAA-Champenoux. 
les plus graves sous peuplement forestier feuillus et résineux. On constate à nouveau des déficits hydriques deux fois plus sévères sous résineux que sous feuillus, et ceci malgré des différences de comportement écophysiologique. La sécheresse de l'année 2003 apparaît bien comme parmi les plus intenses sous feuillus, alors que sous résineux d'autres années ont présenté des contraintes plus fortes. Face à ces intensités de déficit hydrique, tous les types de végétation ne répondent pas de manière identique : certains meurent, d'autres ajustent leur consommation à la restriction d'eau, d'autres encore perdent leurs feuilles précocement pour éviter de se dessécher. Un synthèse récente llustre ces différentes stratégies pow les arbres forestiers (Bréda et al., 2006).

Le déficit hydrique est illustré par la surface hachurée dans l'encart, à partir d"un exemple de réserve utile de $100 \mathrm{~mm}$ : en dessous de $40 \%$ de cette valeur, soit lorsque la réserve du sol chute en dessous de $40 \mathrm{~mm}$ (illustré ici par la ligne pointillée), la croissance des arbres s'arrête. Pour plus de détails, le lecteur se repor"tera à Granier et al., 1999.

Dans un second exemple, la dynamique de l'eau dans le sol sous couvert de résineux est comparée à celle simulée sous couvert prairial et sous culture de mäs (figure 7). Dans le cas des deux couverts végétaux pérennes (résineux et prairie), le dessèchement débute simultanément, mais son intensité diffère très rapidement. Le dessèchement sous prairie est limité par le faible enracinement du couvert végéta] qui n'explore que les 30 premiers centimètres du sol, alors que l'ensemble des $160 \mathrm{~cm}$ du profil de sol est exploré par les racines des arbres. La dynamique hydrique du sol sous maîs est similaire à celle du sol sous peuplement feuiliu en ce qui concerne l'aspect temporel (Fig. 6). Ceci s'explique par l'apparition tandive des surfaces de feuilies, liée à l'implantation au printemps de la culture de mais avec une émergence des plants de mails début maı. Toutefois, l'extraction de l'eau par le maïs reste limitée par son enracinement maximal, qui ne dépasse pas $1 \mathrm{~m}$ de profondeur dans la plupart des cas, et par sa sensibilité au stress hydrique. L'état hydrique du sol à la fin de l'année 2003 est intếressant à comparer (Fig. 7). Que ce soit pour le couvert prairial ou la culture de maïs, le sol a reconstitué sa réserve hydrique dès la fin de l'année 2003, ce qui est aussi quasiment le cas pour le sol sous peuplement de feuillus. En revanche, la réserve en eau reste largement déficitaire en fin d'année sous peuplement de résineux.

\section{6}

\section{Quelle approche pour modéliser la variation de teneur en eau et de volume du sol ?}

L’approche la plus rigoureuse consiste à simuler de façon mếcaniste à la fois le déplacement de l'eau et celui de la phase solide du sol (Angulo et al., 1990a; Garnier et al., 1997). Deux options sont alors possibles. La première possibilité, qualifiée de "méthode eulérienné consiste à décrire le déplacement de l'eaı et du solide par rapport à un repère géométrique cartésien (généralement, l'observateur). L'équation de transport de l'eau est alors nettement plus complexe que dans le cas des sols rigides. La seconde possibilité, appelée 'méthode lagrangienne' consiste à décrire le déplacement de l'eau non plus par rapport à un repère géométrique fixe, mais par rapport à la phase solide du sol (le repère va donc bouger par rapport à l'observateur). On utilise alors des coordonnées dites 'matérielles' à le place des coordonnées spatiales cartésiennes classiques (Smiles et Rosenthal, 1968). Lavantage de cette méthode est que l'équation de transport de l'eau est alors identique à celle utilisée pour les sols rigides (équation de Richards). Les deux méthodes ont été utilisées par Angulo et al. (1990b) pour décrire isinfiltration de l'eau dans une colonne de bentonite.

Une approche plus simple, mais moins rigoureuse, consiste à séparer la simulation du transport de l'eau de celle de la déformation du sol. Le transport de l'eau est alors simulé dans le sol considéré comme rigide. Les variations de teneur en eau du sol sont ensuite traduites en terme de déformation du sol à l'aide de la

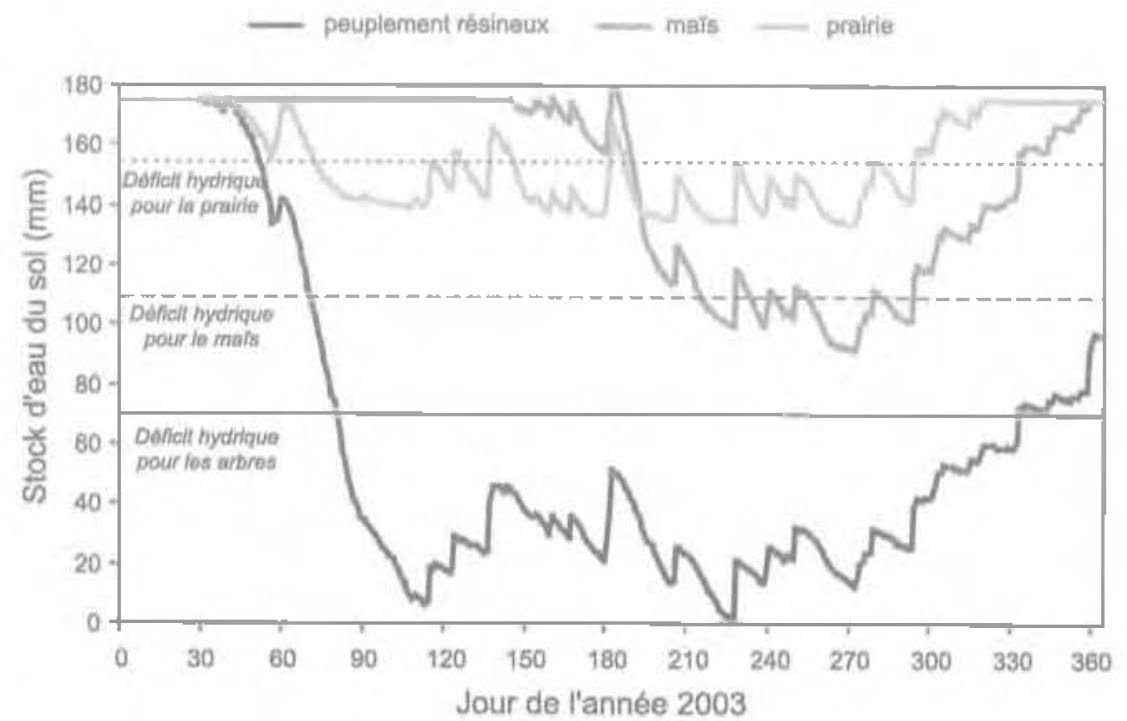

mat $\mathrm{J}$

Évolution simulée de la réserve en eau d'un sol ả forte réserve (175 mm) au cours de l'année 2003 à Grignon, sous peuplement de résineux, sous culture de maìs et sous prairie. Modélisation SWAP (van Dam et al., 1997), données climatiques INFA-Grignon. 
courbe de retrait. L'application de cette méthode au cas présenté en figure 7 permet de calculer le profil de variation maximale de volume par rapport ̀̀ l'état initial (sol à la capacité au champ). Ce profil (Fig. 8) est simplement calculé en utilisant les variations du profil de teneur en eau entre l'état initial et le maximum de dessèchement du sol simulé pour chacune des cutures et en les transformant en variation de volume à l'aide de la courbe de retrait du sol (Fig. 9). En supposant une déformation isotrope du sol, il est alors possible de calculer la variation d'altitude de la surface du sol correspondante : elle est de 8,5 cm sous peuplement de résineux, $4 \mathrm{~cm}$ sous culture de maís et 2 cro sous prairie. De telles valeurs ont pu être effectivement mesurées dans la nature (Bozozlk et Burn, 1960; Cabidoche et Ozier-Lafontaine, 1992; Arnold ef al., 2005).

Bien que cette méthode ne soit pas totalement exacte, elle est cependant d'une précision nettement suffisante pour la plupart des sols rencontrés, y compris les sols argileux (Gamier et al, 1997). Cependant, pour ces sols, la déformation conduit le plus souvent à l'apparition de fissures qui modifient alors profondément le fonctionnement hydrodynamique du sol. Celles-ci peuvent en effet conduire l'eaı qui ne s'infiltre pas à la surface du sol vers la profondeur, de façon très efficace. Le modèle SWAP [van Dam et al. 1997) permet de prendre en compte ce processus, en affectant une partie de la variation de volume du sol due au retrait à la création de fissures. L'eau en excès par rapport à la capacité d'infiltration de la surface du sol est alors directement envoyée dans les fissures (Fig. 10). Ce type de transport rapide de l'eau vers la profondeur a pu être mis en évidence dans le cas de sols argileux déformables (Coulomb et al., 1993; Arnold et a1., 2005).

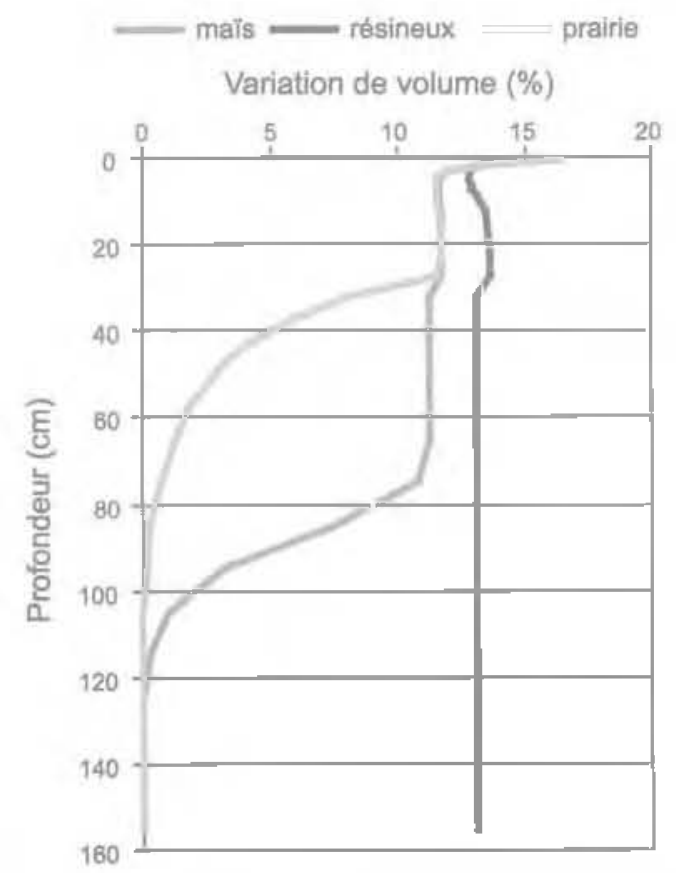

FG. 8 Profil de variation de volume du sol (en \% du volume initial) entre l'état injtial (capacité au champ) et l'élat de dessèchement maximal (le 16 août pour le peuplement de résineux, le 13 aoút pour la culture de maîs, le 29 septembre pour la prairie). La variation de volume est calculée à partir de la courbe de retrait du sol (Fig. 9).

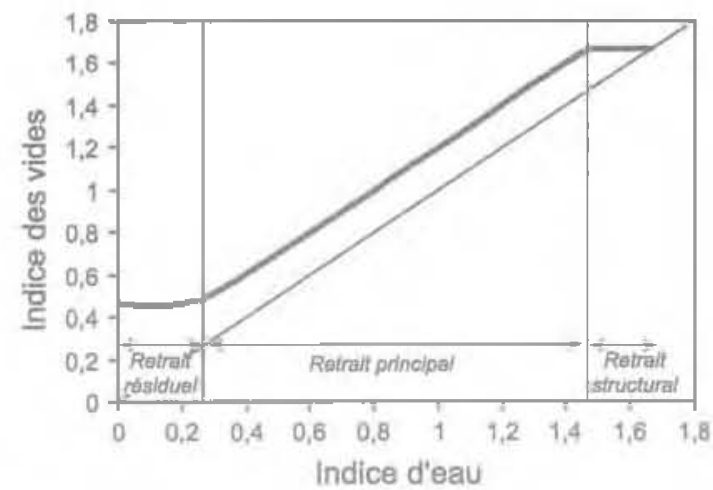

FIG. 9 Exemple decourbede retrait d'un sol argileux. L'indice des vides correspond au volume de la porosité du sol rapporté au volume de sa phase solide. L'indice d'eau correspond au volume d'eau rapporté au volume de la phase solide du sol. Le modèle de Kim et al. (1992) est utilisé pour décrire la courbe.
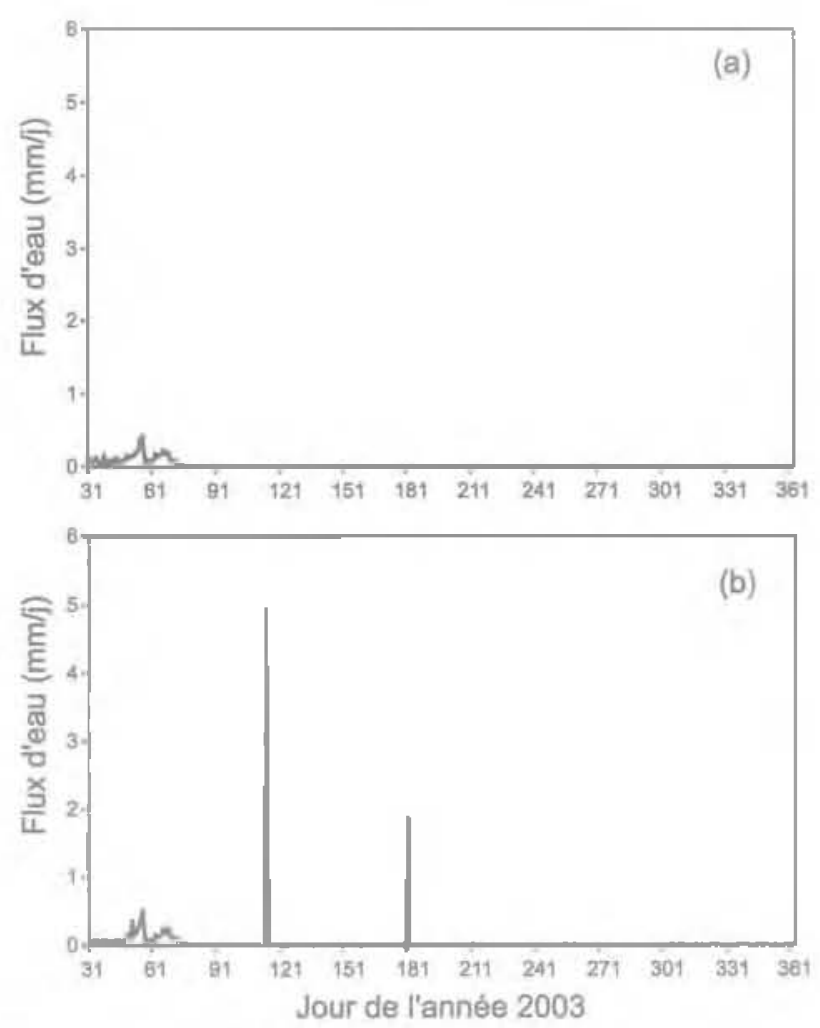

mG. 10 Flux d’eau (en mm/j) à la base du profil de sol (a $160 \mathrm{~cm}$ de profondeur], sous peuplement de résineux, sans prendre en compte la fissuration du sol (a), avec la prise en compte de la fissuration et des flux d'eau dans les fissures (b). Les fortes intensités de pluie conduisent à des flux importants d'eau à la base du sol lorsque les fissures créées par le retrait du sol sont prises en compte, Modélisation SWAP (van Dam et al., 1997), données climatiques INRA-Grignon.

\section{7}

\section{Conclusion - discussion}

La modélisation permet de traduire l'influence de la végétation sur le phénomène de retrait/gonflement des sols de manière quantitative. A ce titre, elle constitue un outil potentiellement intéressant pour la prévision 
des risques géotechniques liés aux aléas climatiques de type sécheresse. Il existe toutefois un certain nombre de limitations qu'un travail de recherche et de développement devrait pouvoir lever à l'avenir.

Larchitecture racinaire des arbres et arbustes utilisés à des fins ornementales ou paysagères, et surtout sa traduction en terme d'efficacité hydraulique effective, c'est-à-dire l'architecture des racines qui puisent l'eau du sol de manière effective, sont encore ma] connues. Cette architecture n'est pas seulement déterminée раг le type d'arbre, mais aussi par les contrairites pédologiques auxquelles il est soumis ('i.e. présence d'un horizon de sol tassé ou engorgé en permanence). L'existence d'une information précise sur la répartition des racines et leur fonctionnement hydrique est essentielle pour une prevision fiable des variations de teneur en eau du sol, et donc de ses déformations.

La sensibilité des sols au retrait/gonflement est délicate à évaluer. Elle inplique la connaissance des types minéralogiques des argiles pouvant être présentes dans le sol. Linformation sur les sols ne doit donc pas seulement comprendre des données permettant de prévoir les propriétés hydriques du sol. Elle doit aussi inclure des informations permettant de prédire sa sensibilité au retrait/gonflement. Des fonctions de pédotransfert permettant cette prëdiction mériteraient d'être développées el testées à l'avenir.

Il faut souligner enfin qu'il n'existe pas à l'heure actuelle de modèle réalisant le couplage complet entre les fonctionnements mécanique et hydrigue du sol et le fonctionnement hydrique de la végêtation. Un tel modèle semble indispensable au développement des recherches sur la prévision du retrait/gonflement des sols. Ce modèle devrait permettre la description de problèmes tridimensionnels, tels qu"on peut les rencontrer en géotechnique (ex. arbres isclés au voisjnage d'un bâttiment). La mise au point d'un tel modèle réclame un travail réellement pluridisciplinaire entre géotechniciens, pédologues et forestiers. Ce travail en collaboration devrait permetire d'elaborer de nouvelles normes, par exemple sur l'ỉmplantation de végétaux au voisinage de structures, plus efficaces dans la prévention des sinistres. Il importe donc de sensibiliser dès à présent la communauté des géotechniciens à l'importance d'une bonne connaissance du fonctionnement du continuum sol-plante-atmosphère pour la prévision du retrait/gonflement des sols.

\section{Bibliographie}

Angulo Fi., Gaudet J.-P., Thony J.-L, Vauclir M. - Conductivité hydraulique d'un inilieu poreux partiellement saturé déformable. I. Principes de déterrnination. Comptes rendus de l'Académie des sciences de Paris, série 2, 310, 1990a, p. 161-164

Angulo R., Gaudet J.-P., Thony J.-L. Conductivitê hydraulique d"un milieu poreux partiellement sature deformable. I1. Résultats expérimentaux. Comptes rendus de l'Académie des sciences de Paris, série 2, 310, 1990D, p. 341-345.

Arnold J.G., Potter K.N., King K.W., Allen P.M. - Estimation of soil cracking aryd the effect on surface runoff in a Texas Blackland Prairie watershed. Hydrol. Process., 19,2005, p. 589-603

Bastet G., Bruand A., Quentin P. Cousin. I. - Estimation des propriétés de rétention en eau des sols à l'aide de fonctions de pédotransfert [FPT) : une analyse bibliographique. Etude et Gestion des Sols, 5 . 1.1998, p. 7-28.

Blanchart E, Achouak W. Albrecht A, Bara kat M. Bellier G. Cabidoche YM. Hartmann C, Heulin Is Larré-Lartouy C, Lälrent $J$ Mahieu $M$, Thomas F, Villemin $G$. Wattea F - Détermínants biologiques de l'agrégation dans les vertisols des Pettes Antilles. Conséquences sur l'érodibilité Étude et Gestion des Sols, 7. 2000, p. 309 328.

Bozozuk M., Burn K.N. - Vertical ground movenents near elm trees. Geotechnique. 10, 1960, p. 19-32.

Braudeau E. - Essai de caractérisation quantitative de l'état structural d'un sol basé stir létude de la courbe de retrait. C. P. Acad. Sct. R, 307, 19, 1998, p. 1933-1936.

Bréda $\mathrm{N}$ - L'indice foliaire des couverts forestiers: mesure, variabilité et rôle fonctionnel. Revue forestière francaise, $\mathrm{n}^{\circ} \mathrm{LJ}, 1999, \mathrm{p}, 135-150$

Bréda N., Granier A, Aussenac G. - Evolutions possibles des contraintes climati ques el consécuences pour la croissance des arbres. Revue forestière trançaise, $I^{\circ}$ LII, 2000. p. 73-90.

Bréda N., Granier A., Alsşenac G. - La sêcheresse de 2003 dans le contexte cljmatique des 54 denières années : aralyse écophysiologique et influence sur les arbres forestiers. Revue forestière frangaise, n'LV., 2. 2004, p. 109-131.

Bréda N., Granier A., Barataud F., Moyne C. - Soll water dynamics in an oak stand. Part I. Soil molsture, water potentials and root water uptake. Plant and Soll, $\mathrm{n}^{\circ} 172$, 1995, p. 17-27.

Bréda N., Granier A., Huc R., Dreyer, E. Temperate forest trees and stands under severp drought: a review of ecophysiological responses, adaptation processes and long-term consequences. Annals of Forest Sciences, vol. 63, $1^{\circ}$ 6, 2006, p. 625644.

Bréda N., Lefévre Y., Badeau V. - Réservoir en eau des sols forestiers tempérés: spécificité et difficultés d'êvaluation. Ia Howille Blanche, $\pi^{\circ} 3$, Forêts et Eau, 2002, p. $25-40$.

Bruand A., Duwai O., Cousin I. Estimation des propriétés de rêtention en eau des sols à partir de la base de données SOL-
HYDRO : ule premièra proposition combinant le cype dhorizon, sa texture et sa densite apparente. Etude et Gestion des Sols, 11, 3, 2004, p. 323-332.

Bruand A., P. Pérez Fernández. O. Duval. Use of class pedotransfer functions based on texture and bulk density of clods to generate water retention curves. Soil Use and Management 19 (3), 2003, p. 232-242.

Cabidoche YM. Ozier-Lafontaine H. 1992 Gestion agricole de l'éu à la parcelle el systèmes de culture irrigués dans la zone carathe. Usage agricole de l'eau. Sixiemes journées hydrologiques de I'ORSTOM. Montpellier, 12-13 sept. 1900. P. Carré éd. ORSTOM, coll. a Colloques at Séminajres 2,1992, p. $15-31$.

Coulomb C. Vachier P. Dever L. - Liilsation de l'oxygène 18 comme traceur in sin de l'infittration : cas d'un sol argilewx drainé C.R. Acad. Sci., 317, série II, 1993, p. 49 55.

Cresswell IL, Coguet Y, Bruand A., McKenzie NJ - The transferability of Australian pedotransfer functions for predicting water retention characteristics of French soils. Soil' Use and Management 22:1, 2006, p. 62-70

Garnier P., Perrier E., Angulo-Jaramillo H., Baveye P. - Numerical model of 3 -dimensional anisotropic deformation and 1 dimensional water tlow in swelling soils. Soll Science, 162 (6), 1997 , p. $410-420$.

Granier A. - Evepotranspiration des forêts. Comptes rendus de l'Académie d'agricuture de France, 82, 1996, p. 119-132. 
Granier A., Bréda N., Biron P., Villette S. A lumped water balance model to evaluate duration and intensity of drought constraints in forest stands. Ecologica? Modelling, 116, 1999, p. 269-283.

Granter A., Badeau V., Bréda N. - Modélisation du bilan hydrique des peuplements forestiers. Revue Forestière française, $n^{\square}$ XLVII, 1996, p. 59.68.

Kim D.J., Vereecken H.., Feyen J., Boels D. Bronsw.jk J.J.B. - On the characterization of properties of an unripe marine clay sofl. 1. Shrinkage processes of an unripe clay soil in relation to physical ripening. Soil Science, 153 (6), 1992. p. $471-481$.

Smiles D.E., Fosenthal M.J. - The movement of water in swelling materials. Australian Journal of Soll Research 6, 1968, p. 237-248.

Tessier D., Bruand A., Lebissonnais Y, Dambrine E - Qualité chimique et physique des sols: Variabilité spatiale et évolution. Etude et Gestion des Sols, 3 , 4,1996, p. 229-244. van Dam J.C., Huygen J., Wesseling J.G., Feddes R.A., Kabat P. van Walsum P.E.V., Groenendijk P, van Diepen C.A. Theory of SWAP version 2.0. Simulation of water flow, solute iransport and plant growth in the Soil-Water-AtmospherePlant environment. Report 71, Depart ment Water Resources, Wageningen Agricultural University, Technical Documert 45, DLO Winand Staring Centre, Wageningen, 1997. 